\title{
Sustainable space optimization and function versatility through mass displacement
}

\author{
S. S. Zubir ${ }^{1}$, Q. Norhisham ${ }^{1,2} \&$ Y. Rahman ${ }^{2}$ \\ ${ }^{1}$ Centre of Studies for Architecture, \\ Faculty of Architecture, Planning and Surveying, \\ Universiti Teknologi MARA, Malaysia \\ ${ }^{2}$ MDRXA Studio, Malaysia
}

\begin{abstract}
Despite the current brouhaha over energy issues, the problem of sustainability is not just limited to matters of earth, water, air and environment but also should include the functional program of buildings. Therefore, architectural design must be approached creatively to meet future demands and challenges. The idea of creating large undefined flexible space is no longer a sustainable approach. This design escapade attempts to present an alternative to the current event space scenario with optimized performance of the space through juxtaposition in a proposed Institute for Mental Athletics. By creating a structure that embodies a number of programs, a single structure can truly be used all year round with minimal or no physical alteration at all. This creates a new paradigm in thinking of sustainable space. Instead of having four different event spaces to host different functions, a singular geometry that is capable of performing the same task will save space, materials and also operational cost. The proposed design of the hedron evolves but stays true to the notion that it is an object that can be manipulated with ease according to the user's demand. The structure and service has to be independently integrated to ensure it functions efficiently just like an object. In order to appreciate the essence of spatial coherency in the hedron, one must understand how each plane of the hedron plays a different complimentary role. The complexity between the profile relationships goes beyond physical form. They function as independent surfaces, but also work together. This idea can be brought forward into other event space design that requires versatility to achieve a sustainable program.

Keywords: flexible space, architecture, juxtaposition, sustainable space, hedron, versatility.
\end{abstract}




\section{Introduction}

The gaming industry is emerging as one of the most popular entertainment medium in Malaysia. For the past five years, the gaming community has actively organized events, tournaments and exhibitions [1]. Gaming events require a set of unique spatial quality in order to be successfully conducted. Therefore, game event organizers will usually resort to rent large spaces such as convention halls to host these events.

The blunt and characterless event spaces result in sub-par event quality. The generalization and lack of character diversity of existing event halls result in compromised visitor experience and high cost of altering the space to suit the event's requisite [2].

Versatile event space in the past works solely on the basis of whether the space is large enough to accommodate a program or an event, but they do not satisfy the need of unique physical space attributes of a gaming event. It is not that all programs demand an enormous amount of space, but rather a different characteristic of surface profile and/or wall and ceiling features that satisfies the gaming event program spatial need. In order to achieve such quality, the idea of implanting space with artificial intelligence and mechanics that allow them to morph, alter and respond to the user needs must be explored. A new principle based on mass displacement will be formulated to achieve space versatility and detach from the idea of flexible space that is only based upon shrinking and expansion of space volume [3].

The manifesto sets to push forward technologically, refine and reconfigure the idea of spatial versatility and program juxtaposition by physical manipulation. The result of the manifesto will reveal the challenges, potential, and future applications of such spatial concept that can benefit the future building typology that requires such flexibility of a space and ability of multi-purpose programs superimposed in one volume.
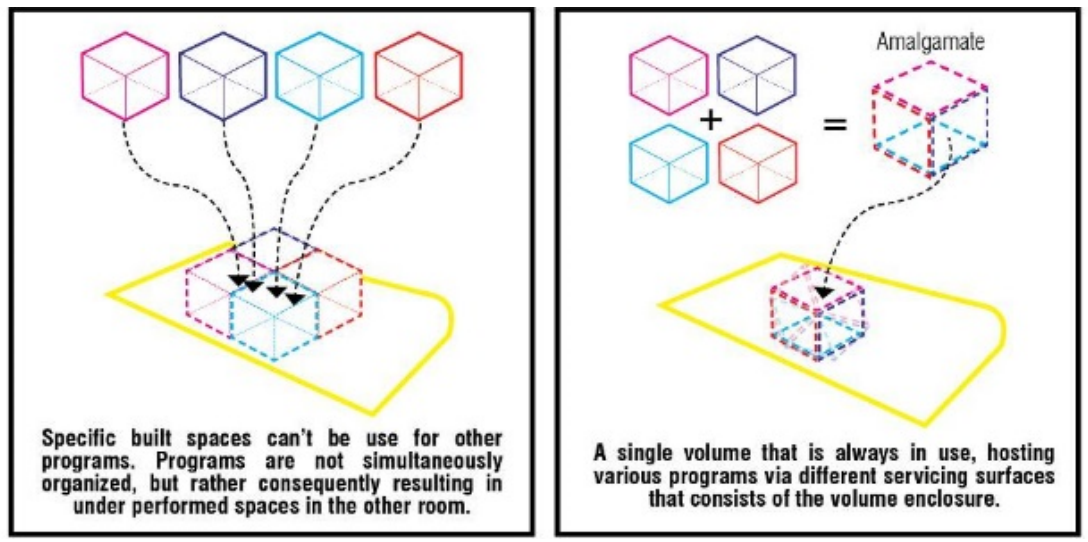

Figure 1: The idea of shifting from multiple volumes to host multiple events into one volume that hosts multiple programs. 
To achieve the goal of this design research, the study will reveal the architectural perimeter of gaming programs that will be chosen to be moulded into a spatial volume. The perimeter will be an array of architectural functions that include the services, construction and volume profile for the combined programs. This explorative study will culminate into a design proposal.

\section{Juxtaposition}

The proposed design evolves but stays true to the notion that it is an object like architecture, whereby it can be manipulated with ease according to the user's demand. In order to appreciate the formalism of spatial coherency in the hedron, one must understand how each plane of the hedron plays a different complimentary role. The complexity between the profile relationship goes beyond physical form. They function and perform as individual surfaces, but also work together.

The manifesto embodies the idea of space as a constant flowing commodity that is only defined by its enclosure, but space nevertheless, flowing. The poetry of movement in architecture has always intrigued architects and users alike. As the world becomes more and more demanding, we must manage our most precious commodity more effectively, space.

Space is most precious the way they come, raw and undefined. As architecture defines these raw spaces into specific commitment, we can no longer see the potential of the space as if it were left free [4]. These scenarios are a challenge that this manifesto accepts, by exploring the spatial potential, beyond its conventional inception. By creating a structure that embodies a number of programs, a single structure in the form of the polyhedron can truly be used all year round with minimal or no physical alteration at all.

This creates a new paradigm in thinking of sustainable space. Instead of having four different event spaces to host different events, a singular geometry that is capable of performing the same task will save space, materials and also maintenance cost [5].

\section{Infrastructure}

The discussion of the new type of event space can start by first, discarding the traditional notion of architecture as the closure of space. Instead of architecture becoming a temporary space container, rethinking architecture as a host of space that works just like an infrastructure network in a city that channels resources and commodity where needed, and how much is needed.

This theory deemed architecture as an infrastructure that forms spatial quality, quantity and aesthetics by following these 2 rules:

\section{Rule 1}

Space is treated as a flowing commodity. Not static, not bounded. Space is fragile and unstable. Subject to change and inconsistency as external forces affect them. 


\section{Rule 2}

Architectural elements as infrastructure that channels space where and how much it is needed. Mimicking the role of road and rails in the city, space can be transferred through these infrastructural networks. Space can be expanded, shrink and lifted or grounded.

This theory suggests architecture consists of tools to frame space as a flowing commodity. The theory encourages architecture not as a static space enclosure, instead, build infrastructure that gives the user the capability to form or define space according to their need.

Imagining a building as a complex city, the architecture will become the vital infrastructure that changes as it serves its users, thus creating a dynamic range of its uses and its future potential. Opening up possibilities of space makes them insecure and unstable. But by doing so, we unlock and release space from its singular commitment, and open up endless potentials and possibilities in the future. Due to the new nature of these spaces, there are a number of external forces or scenarios that affect creation and usage of these spaces.

\section{Theories of pre-juxtaposition}

\subsection{Theory 1: large container versus unique profiles}

The large container can host S, M, L, and XL programs in the same spatial environment. (Limited to programs similar in nature) This is the conventional interpretation of flexible space, which will be challenged in the manifesto.

The research attempts to re-introduce the unique surface profile as the real flexible space. But unfortunately, these unique profiles are rarely fused together to become a space enclosure. They are usually used individually due to extremely unique program nature, which they host. Unlike the large container, they can't be partitioned to host different or even multiple programs [6].

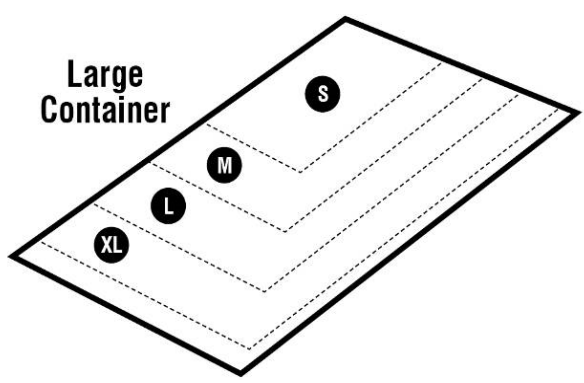

Figure 2: The figure suggests the ability of a large contained space to host multiple programs, thus labelled as flexible.

Thus spatial juxtaposition of these unique profiles is the key to unlock the true potential and possibility of architecture to escalate from an enclosure to be infrastructure-like in nature. Only then, the same volume of space can give amplitude of experience over and over again. 


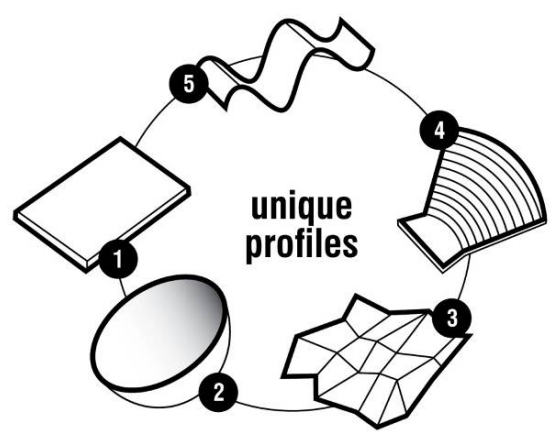

Figure 3: From 1 to 5: Flat profile/vector based surface, bowl profile/ double curvature surface, fragmented profile/multi vector surface, stepped/terraced profile/slanted surface and undulating profile/ tangent based surface.

\subsection{Theory 2: mass displacement as method for spatial manipulation}

1. Rotate - a geometry/solid/volume can easily be manipulated by rotation. Note that, taking a flat surface as a datum, the servicing surface area changes as the rotation degree differs.

2. Slide - taking a portion of the volume in a direction to create a new spatial quality. Opening up to the sky, or creating a unique profile (which is determined by the cutting geometry).

3. Hedrons - the geometry that defines the hedrons are varied from triangles, pentagons, and so on. The consequence of having many surfaces to build a hedron is that each surface would be small. This relationship has to be balanced between the numbers of programs to be integrated within the area needed for each program.
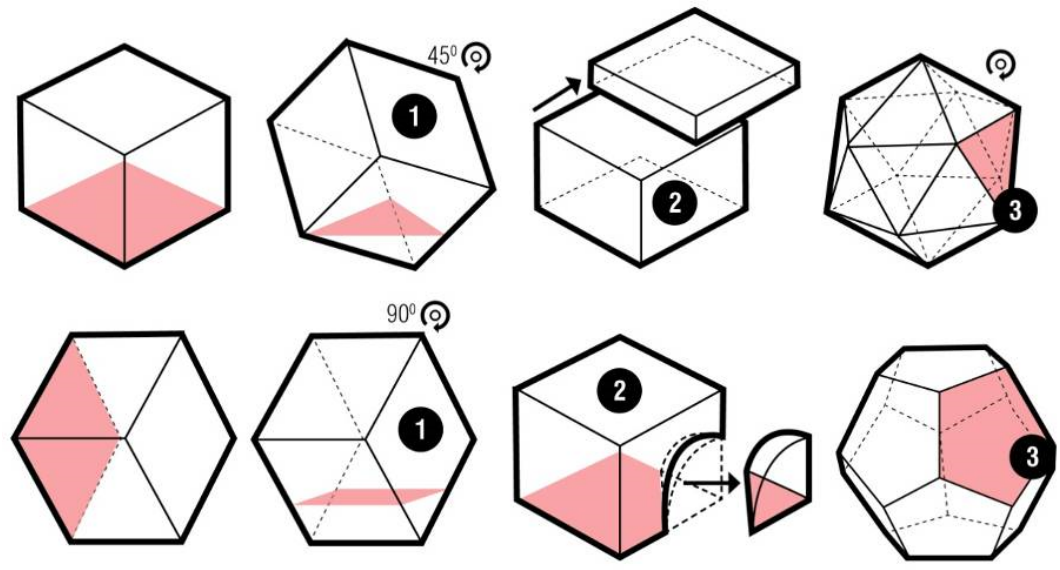

Figure 4: Possible mass displacement of a single cube mass. 
The idea of architectural space has always started from a point, line, plane and eventually volume. Through the formation of these elements, the plane's potential has been domesticated by assigning specific functions such as floor (servicing surface), walls (vertical enclosure), and ceiling/roof (horizontal enclosure) [7].

\subsection{Theory 3: objects like architecture}

The creation of objects has always trailed closely with the advancement of technology. In order for architecture to behave like an object, it must suggest a notion where it must embodied a few characteristics of an object such as:

1. Free to be used in any way the user wants: flexible and within the range of capability. (Architecture will have to be more users responsive and designed from ground up to host multiple programs).

2. Can function independently/almost independently without constant feed of energy or input. (The services in the architecture must be efficient and portable, similar in nature to trains or airplanes. The services must not be the strap that limits the usage of an architectural space).

3. Mobile and easily customized. (Architecture that is able to be freely orientated moved and by minimal input transformed into a customized space).

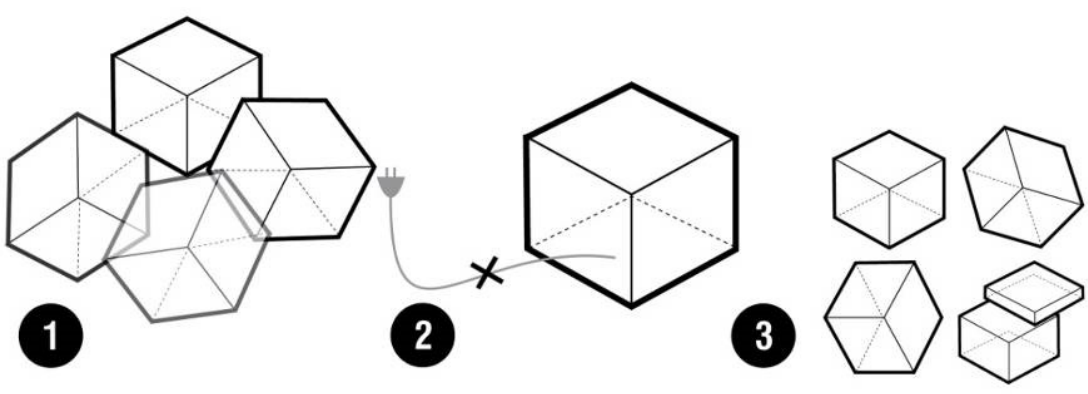

Figure 5: Architecture that embodies object characteristics.

\section{Scenario of events in juxtaposed spaces}

\subsection{Scenario 1: an analogy - events are viruses that spread}

Events in The Institute will stimulate a virus spreading in a biological matter. The virus will not only infest on the host, but also spread and infect other parts of The Institute. Since events in The Institute are vaguely bounded, an event starting in one space can easily spur out of its initial ignition point into other spaces. These endless possibilities of virus programming in The Institute makes instability a unique feature that injects a surreal environment in The Institute. Yet, there are some spaces that would be immune to any changes, and deter off any inconsistencies in order to maintain balance. 


\subsection{Scenario 2: circulation is celebrated}

Circulation is celebrated as the prime space, as it is the only space that is stable in The Institute. The circulation will most likely to be the in-between spaces that pit together two or more programs, therefore the circulation will be the vein of The Institute that brings out activities in the space beyond their initial designated boundary. These flowing strings of events can generate interest of users in other spaces through demonstration in the circulation spaces.

\subsection{Scenario 3: intersections of programs, are point of ignition}

A program's activity will affect neighbouring programs and create an overlapping effect of activities. The most interesting activity in The Institute will not only be the activities happening in the space, but the overlapping areas that will create a form of hybrid activity. For example, a gaming area that overlaps with a cafeteria will form an interesting result as to how the user uses the overlapping program's space. The idea is to create an unstable area where the user can impose their own activities, thus creating a new hybrid program in the intersected area.

\subsection{Scenario 4: operate/shutdown autonomously and collectively}

The components of The Institute can operate autonomously or/and together. Despite instability, the whole complex hosts programs with different users, operation time and privacy gradient. These components of programs must be able to be shut off from other parts of The Institute (if needed) and still allow other components to function as usual without any disturbance. When all components are in operation, The Institute will benefit from complimentary nature of each component working together to enhance each other's spatial experience and efficiency.

\section{Juxtaposing process}

\section{A. Geometry appraisal}

Tetrahedrons are built out of a collection of four (4) same-sided triangles. This 3D geometry has the best structural integrity in all possible directions as the geometry is rotated.

B. Closure to surface ratio

The tetrahedron is the best geometry suited for the project. All enclosed surfaces of the volume can be used to host programs, creating $100 \%$ value for material usage to build the event hedron.

\section{Manipulation principle}

The hedron has a triangle floor plate geometry that can be manipulated. The floor profile must at least have one contact point on each line to make up the whole triangle.

D. Trimming surfaces

The hedron triangle unusable corner surface is trimmed off to reduce overall size and increase material per usable area efficiency. 
A

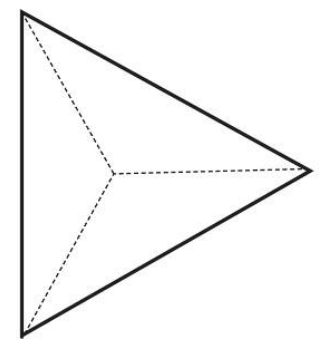

B

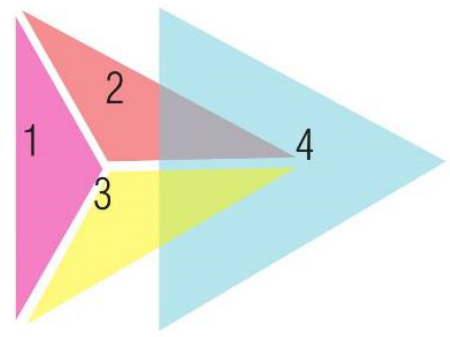

Figure 6: A illustrates the tetrahedron in its native form and B shows the Tetrahedron has four triangle surfaces with equal surface area.

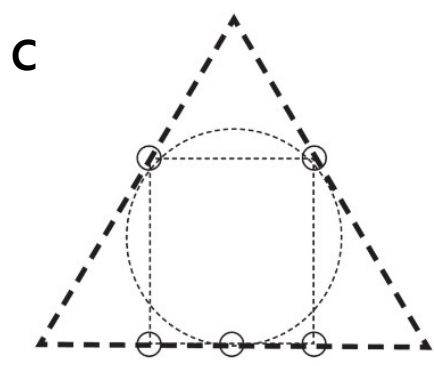

D

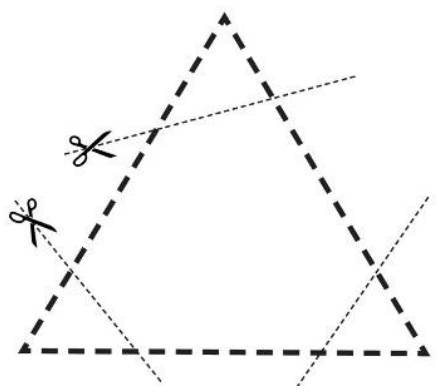

Figure 7: $\mathrm{C}$ is an example of triangle manipulation into a circle and a rectangle whereas D illustrates the selective geometry trimming, reducing underutilized area and increasing usable area in the triangle geometry.

\section{E. Stamping profile}

Once the geometry has been redefined, the surface can be stamped to suit specific profiles that can allow the surface to host unique events.

F. Changing surface/orbital rotation (mass displacement)

Each surface will have their own special designated program to host. The surface will function as floor, walls and ceiling depending on the hedron orientation. The profile on each plane will aid and compliment other plane functions. In order to ensure efficient phasing mode, the hedron is mounted on two rings that allow it to be rotated $360^{\circ}$, thus mimicking the orbital movement. These movements cater the floor profile shifts in a safe and fast method without help from external forces. The swing rings act as the mediator of these unique planes. They serve as the main mechanism that rotates the whole structure on both $\mathrm{x}$ and $\mathrm{y}$-axis to mimic an orbital rotation. 
E

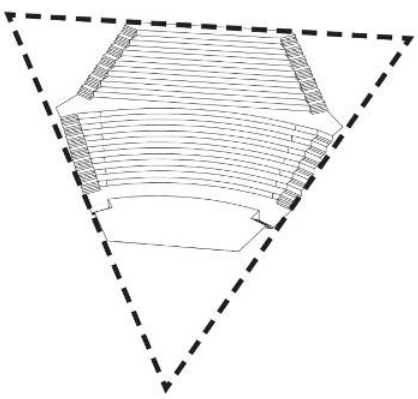

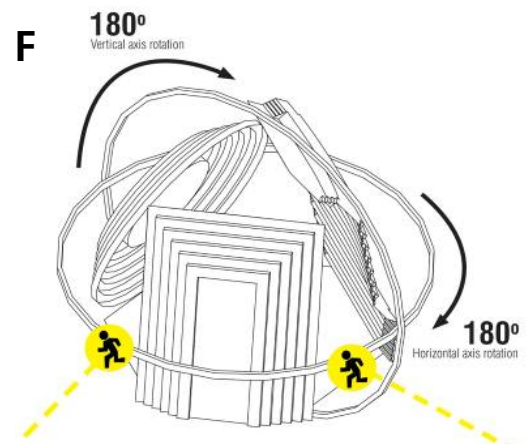

Figure 8: $\quad$ E is a flat surface, which has been stamped into an auditorium profile to enable it to host any event needing such profile. F is a conceptual illustration on how the different surface profiles can be combined into a 3D geometry with two axial rotations to change the unified geometry base surface.
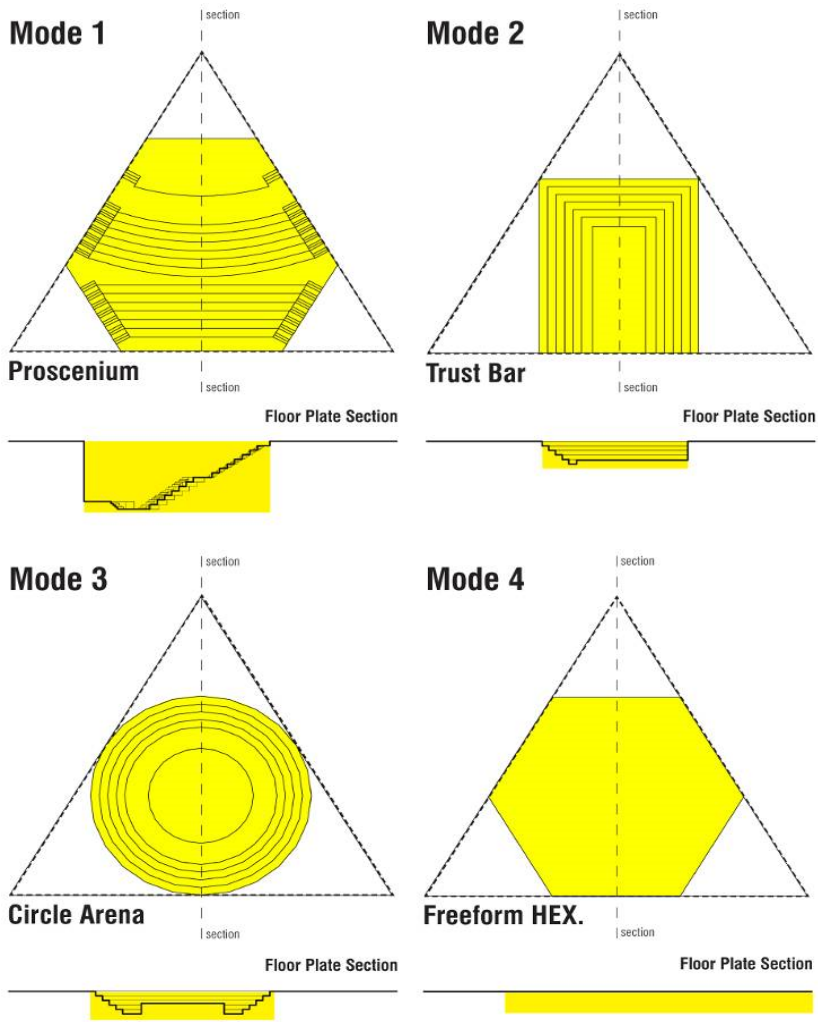

Figure 9: Mode 1 to 4 indicate the initial configuration phases that established the formulation of the hedrons final geometry and sectional profile. 


\section{Conclusion: architecture as an experiment}

The Institute for Mental Athletics is an event complex, research and training center that propels the development of the gaming industry in Malaysia. The Institute is designed to be in constant state of instability. The spaces are in constant and ongoing change creating a multitude of experiences to the users. The complex is designed to be sustainable beyond efficient resource consumption, as it is to be able to host programs that might be held in the future.

The design breaks into separated but integral components that work autonomously from one another, but at the same time complement each other and can work together. These components are, The Institute, The Event Hedron, The Living Room and The Offices. The complex embodies the spirit of dual nature in spatial configuration that can morph and respond to the multiple event types that would be held by the operators.

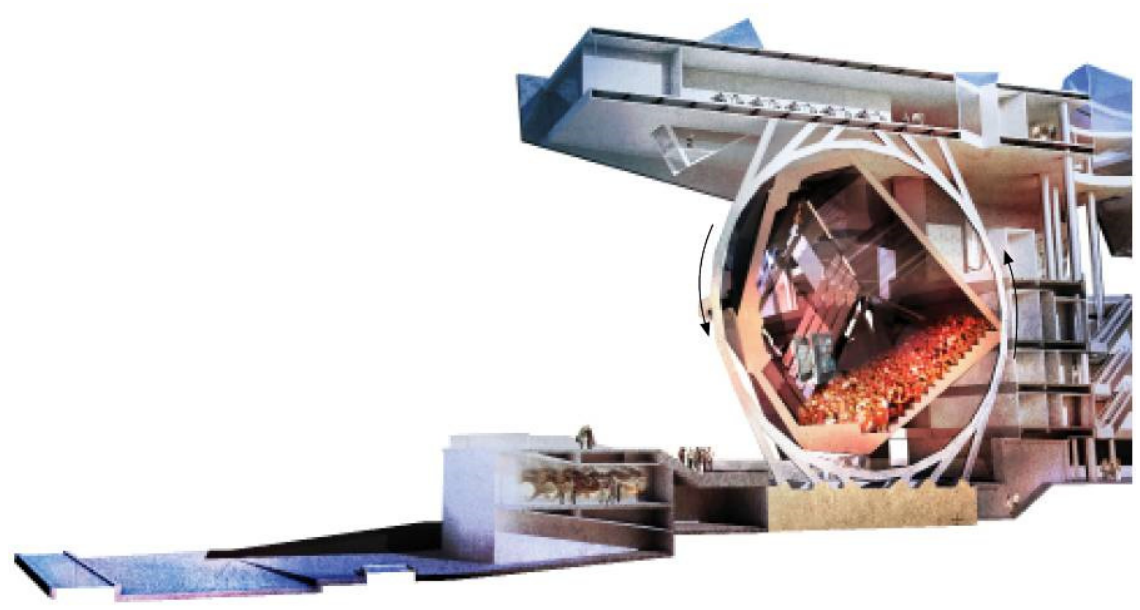

Figure 10: Cross section of the Event Hedron exposing the optimum potential of a volume to host different events based on distinct sectional characteristics of the surfaces.

The Event Hedron has surfaces with their own specially designed program to host. The surfaces will function as floor, walls and ceiling depending on the hedron's orientation. The profile on each plane will aid and compliment the other planes functions. In order to ensure efficient phasing mode, the hedron is mounted on to two rings that allow it to be rotated $360^{\circ}$, thus mimicking the orbital movement. These movements cater the floor profile shift in safe and fast method without any external forces. The swing ring acts as the mediator of these unique planes. They serve as the main mechanism that rotates the whole structure on both $\mathrm{x}$ and $\mathrm{y}$-axis to mimic an orbital rotation. 
The concepts of electrodynamics are mainly related on levitation by repulsive and attractive force between magnetic fields. Both forces can be manipulated and controlled to rotate the Hedron mass to a desired configuration. Maglev technology allows the mass of the hedron to be fully elevated and suspended in the Hedron Ring. The Hedron mass can be manipulated by the directional pull and push of magnet forces without any friction.

Building a space consumes a lot of resources and energy, thus the responsive way to utilize the spent energy must be rethought. This research explores the optimum potential of a space to serve more than just one function to have a multiplying effect on the energy and resources spent. Research in design and construction of spaces with reference to this model will not only reduce the resources and material needed for buildings due to integration of function but it will contribute to a new paradigm shift of creative thinking in sustainability.

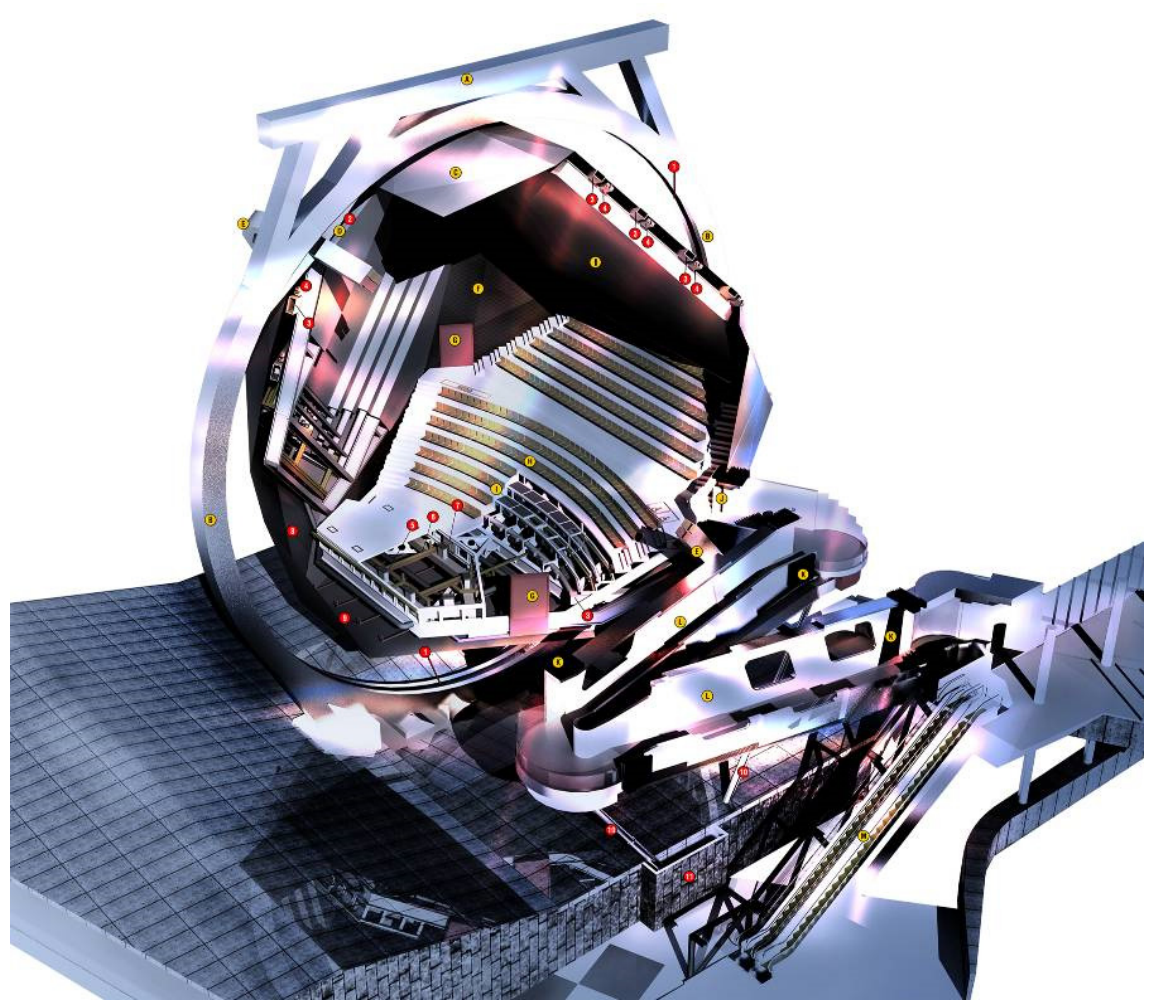

Figure 11: Cut away axonometric of the Event Hedron with its entrance jet way that accommodates the entrance at different levels for different flooring profiles. 


\section{References}

[1] Jane McGonigal, Reality is Broken: Why Games Make Us Better and How They Can Change the World. Vintage Book, London, 2011.

[2] Jane McGonigal, Engagement Economy: The future of massively scaled collaboration and participation. Institute for the Future, 2008.

[3] Anderson, Stacy, Nobbs, Karinna, Wigley, Stephen M. and Larsen, Collaborative Space: An Exploration of the Form and Function of Fashion Designer and Architect Partnership, 2010.

[4] Max Warren, Architecture in Motion: Change We Can Believe In. Unitec New Zealand, 2010.

[5] PRADA (2009) http://www.prada-transformer.com/ as seen on 4th June 2012.

[6] Konstantinos-Alketas Oungrinis (2001) 'Sensponding' Architecture: Towards A Holistic Approach to Transformable Design Aristotle University of Thessaloniki, Polytechnic School.

[7] Michael Malofiy (1998) Order and Flexibility: Their Coexistence as Architectural Principle Virginia Polytechnic Institute. 